\title{
Biopharmaceutical characterisation of ciprofloxacin-metallic ion interactions: Comparative study into the effect of aluminium, calcium, zinc and iron on drug solubility and dissolution
}

\author{
ALEKSANDRA STOJKOVIĆ* \\ LIDIA TAJBER ${ }^{2}$ \\ KRZYSZTOF J. PALUCH ${ }^{2}$ \\ ZORICA DJURIĆ ${ }^{1}$ \\ JELENA PAROJČIĆ \\ OWEN I. CORRIGAN ${ }^{2}$ \\ ${ }^{1}$ Faculty of Pharmacy \\ University of Belgrade \\ 11000 Belgrade, Serbia \\ 2 School of Pharmacy and Pharmaceutical \\ Sciences, Trinity College \\ University of Dublin \\ Dublin 2, Ireland
}

Accepted November 13, 2013

\begin{abstract}
Ciprofloxacin bioavailability may be reduced when ciprofloxacin is co-administered with metallic ion containing preparations. In our previous study, physicochemical interaction between ciprofloxacin and ferrous sulphate was successfully simulated in vitro. In the present work, comparative in vitro ciprofloxacin solubility and dissolution studies were performed in the reactive media containing aluminium hydroxide, calcium carbonate or zinc sulphate. Solid phases collected from the dissolution vessel with aluminium hydroxide, calcium carbonate and zinc sulphate were investigated for their properties. The results obtained indicate that different types of adducts may form and retard ciprofloxacin solubility and dissolution. In the case of aluminium, no phase changes were observed. The solid phase generated in the presence of calcium carbonate was identified as hydrated ciprofloxacin base. Similarly to iron, a new complex consistent with $\mathrm{Zn}\left(\mathrm{SO}_{4}\right)_{2}(\mathrm{Cl})_{2}$ (ciprofloxacin $)_{2} \times n \mathrm{H}_{2} \mathrm{O}$ stoichiometry was generated in the presence of relatively high concentrations of ciprofloxacin hydrochloride and zinc sulphate, indicating that small volume dissolution experiments can be useful for biorelevant dissolution tests.
\end{abstract}

Keywords: ciprofloxacin, dissolution, drug interactions, solubility, solid state characterisation

Reduced ciprofloxacin absorption from formulations containing ciprofloxacin hydrochloride when co-administered with metallic ion containing preparations has been reported in a number of in vivo studies (1-9). While formation of a nonabsorbable complex has been postulated as the interaction mechanism $(1,10-12)$, some authors comment that physicochemical factors such as solubility may also play a role (4). Several authors report that ciprofloxacin hydrochloride tablet dissolution is retarded in the presence of metallic compounds, indicating that dissolution might be used as an in vitro tool for

\footnotetext{
* Correspondence; e-mail: sandric26@gmail.com
} 
drug interaction simulation $(11,13)$. In our previous study $(14)$, a poorly soluble complex with a 2:1 ciprofloxacin to iron ratio was isolated from media used for ciprofloxacin hydrochloride solubility and dissolution studies in the presence of ferrous sulphate. Biorelevant dissolution data reflecting ciprofloxacin-iron interaction were obtained in a small volume dissolution apparatus (14). In the light of the known formation of metal ion complexes of $\mathrm{Ca}, \mathrm{Zn}$ (15) and $\mathrm{Al}$ (16) with ciprofloxacin, it was hypothesised that such complexes may form an impact on ciprofloxacin hydrochloride solubility and dissolution. In the present study, ciprofloxacin hydrochloride solubility in water and reactive media containing different amounts of aluminium, calcium and zinc compounds, as well as comparative ciprofloxacin tablet dissolution studies in the presence of different metallic ions have been performed. In order to identify the nature of physicochemical interactions, if any, characterisation of the solid phases collected from the dissolution vessel following ciprofloxacin tablet dissolution in water in the presence of aluminium hydroxide, calcium carbonate and zinc sulphate was made.

\section{EXPERIMENTAL}

\section{Materials}

Ciprofloxacin hydrochloride tablets (Marocen $500 \mathrm{mg}$, Hemofarm, Serbia) were purchased commercially. Marocen tablets contain $500 \mathrm{mg}$ of ciprofloxacin as the active ingredient while the other excipients are: microcrystalline cellulose, crospovidone, hydroxypropyl methylcellulose, macrogol 4000, magnesium stearate, maize starch, colloidal silicon dioxide and titanium dioxide (E 171).

Ciprofloxacin hydrochloride salt was kindly donated by Hemofarm, Serbia. The investigated metallic ion containing compounds were: aluminium hydroxide (Fluka, Buchs, Switzerland), calcium carbonate (Centrohem, Stara Pazova, Serbia), zinc sulphate, heptahydrate (Sigma-Aldrich Chemie $\mathrm{GmbH}$, Steinheim, Germany) and ferrous sulphate, heptahydrate (Centrohem, Stara Pazova, Serbia).

\section{Solubility}

Ciprofloxacin hydrochloride solubility in water, without and with addition of different amounts of investigated metallic compounds (aluminium hydroxide, calcium carbonate, zinc sulphate and ferrous sulphate), was determined as described earlier by Parojčić et al. (14). All determinations were performed at ambient temperature $\left(25^{\circ} \mathrm{C}\right)$. Samples were continuously shaken on a laboratory shaker (Unimax 1010, Heidolph, Schwabach, Germany) for six hours, then centrifuged and filtrated through the cellulose nitrate membrane filter (pore size $0.45 \mu \mathrm{m}$; Millipore, USA), appropriately diluted and assayed by UV-spectrophotometry (UV-Vis spectrophotometer Evolution 300, Thermo Fisher Scientific, Madison, USA) at $276 \mathrm{~nm}$. Sample $\mathrm{pH}$ values were monitored (pH meter-HANNA 9321, USA). All the solubility studies were performed in triplicate. 


\section{Dissolution}

Dissolution studies were performed at $37 \pm 0.5^{\circ} \mathrm{C}$ using the mini paddle dissolution apparatus (Erweka DT 700, Heusenstamm, Germany) at $50 \mathrm{rpm}$, using $50 \mathrm{~mL}$ of water as dissolution medium, without and with addition of different amounts of aluminium hydroxide, calcium carbonate, zinc sulphate and ferrous sulphate. To evaluate the influence of different cations on ciprofloxacin dissolution, the investigated metallic compounds were added to each vessel concomitantly with the ciprofloxacin tablet in different amounts, corresponding to those used in solubility studies. Dissolution studies in water were performed using the amounts of aluminium hydroxide $(1800 \mathrm{mg})$, calcium carbonate $(3400$ $\mathrm{mg}$ ) and ferrous sulphate heptahydrate $(496 \mathrm{mg}$ ) 'equal' to the doses administered in vivo in relevant interaction studies $(2,4)$. Use of the same doses of aluminium hydroxide, calcium carbonate and ferrous sulphate as in clinical studies $(2,4)$ was aimed at in vitro simulation of ciprofloxacin-metallic cation interactions observed in vivo. Amounts of zinc sulphate used (500, 1000 and $2110 \mathrm{mg}$ ) corresponded to the level of this salt used in the solubility study. All experiments were performed in triplicate. Where a solid phase was formed during dissolution studies, it was collected at the end of the dissolution run and dried at room temperature for further characterisation.

\section{Solid state characterisation}

Solid phases collected after the dissolution studies of ciprofloxacin hydrochloride tablets in the presence of aluminium hydroxide, calcium carbonate and zinc sulphate were characterised using powder X-ray diffraction (XRD), Differential Scanning Calorimetry (DSC), Thermogravimetric Analysis (TGA) and Fourier Transform Infrared Analysis (FTIR).

Powder samples were studied by the XRD technique using a Miniflex II (Rigaku, Tokyo, Japan) Desktop X-ray diffractometer with an Ilaskris cooling unit. The tube output voltage employed was $30 \mathrm{kV}$ and tube output current was $15 \mathrm{~mA}$. A Cu-tube with $\mathrm{Ni}$-filter suppressing $\mathrm{K} \beta$ radiation was used. The scanning angle ranged from 5 to $40^{\circ}$ of $2 \theta$ scale at a step size of $0.05^{\circ}$ per second in each case (17).

Differential scanning calorimetry (DSC) experiments were conducted using a Mettler Toledo DSC $821^{\mathrm{e}}$ (Mettler Toledo Ltd, Zurich, Switzerland) with a refrigerated cooling system (LabPlant RP-100). Nitrogen was used as a purge gas. Aluminium sample holders were sealed with a lid and pierced to provide three vent holes. Sample volume was sufficient to provide proper contact between the powder and the bottom of the pan, and sample mass was $5 \mathrm{mg}$ (17).

Thermogravimetric analysis (TGA) was performed using a Mettler TG 50 (Greifensee, Switzerland) module linked to a Mettler MT5 balance in the furnace under nitrogen purge. Sample masses were between 5 and $12 \mathrm{mg}$ and were placed into open aluminium pans. A heating rate of $10^{\circ} \mathrm{C} / \mathrm{min}$ was implemented in all DSC and TGA measurements. Analysis was carried out and monitored by the Mettler Toledo STAR ${ }^{\mathrm{e}}$ software (version 6.10) with a Windows NT operating system. The unit was calibrated with indium and zinc standards (17). 
FTIR spectra were recorded on a Fourier Transform Infrared Nicolet Magna IR 560 E.S.P. spectrophotometer coupled with a MCT/A detector (Thermo Electron Corporation, Waltham, MA, USA). Data were acquired with the Nicolet software Omnic (version 4.1). Sixty-four scans of symmetrical interferograms were averaged and the spectrum was calculated from 650 to $4000 \mathrm{~cm}^{-1}$ at $2 \mathrm{~cm}^{-1}$ spectral resolution. A KBr disc method was used with $1 \%(\mathrm{~m} / \mathrm{m})$ sample loading. $\mathrm{KBr}$ disks were prepared by direct compression under 8 bar pressure for 1 minute (17).

\section{Quantification of zinc by inductively coupled plasma-mass spectrometry (ICP-MS)}

Determination of the zinc content in the sample collected from the dissolution vessel in the presence of $2110 \mathrm{mg}$ zinc sulphate was performed using ICP-MS (Palo Alto, CA, USA). A known mass of the sample was placed in a digestion vessel and then treated with $69 \% \mathrm{HNO}_{3}$ and $30 \% \mathrm{H}_{2} \mathrm{O}_{2}$. The vessel was sealed and heated in a microwave digester operating at $1000 \mathrm{~W}$ for 20 minutes at $200{ }^{\circ} \mathrm{C}$. The sample was then diluted with deionized water and the digest was analysed with ICP-MS Varian 820 (Palo Alto, CA, USA).

\section{Quantification of chloride and sulphate ions by photometry}

Quantification of chloride and sulphate ions by photometry was performed as described earlier by Parojčić et al. (14).

\section{Statistical analysis}

Differences between the amounts of ciprofloxacin dissolved in the presence of investigated metallic compounds were evaluated by one-way ANOVA (SPSS Statistics 20, USA). Differences between the groups (i.e., the amount of ciprofloxacin hydrochloride dissolved in the presence of aluminium hydroxide, calcium carbonate, ferrous sulphate or zinc sulphate) were compared using the post-hoc LSD test. Probability of $p<0.05$ or less was considered significant. Double-sided confidence interval (i.e., $95 \%$ ) was used for statistical analysis.

\section{RESULTS AND DISCUSSION}

\section{Literature overview of ciprofloxacin bioavailability}

Ciprofloxacin hydrochloride is rapidly absorbed after oral administration. Results of a remote control capsule study (25) revealed that ciprofloxacin is predominantly absorbed in the duodenum and upper jejunum, with $78 \%$ of dose absorbed from this gastrointestinal segment. Such data suggest the existence of a narrow absorption window in the proximal intestine and indicate that potential interactions occurring after drug ingestion may strongly affect its bioavailability.

A literature survey of ciprofloxacin bioavailability, when administered with and without different metallic ion containing preparations, was undertaken. The overview of 
the available data is presented in Fig. 1 where the first column represents ciprofloxacin absolute bioavailability data obtained from a number of studies (18-23) while other columns represent relative bioavailability (i.e., ratio of $A U C$ S relevant to the interaction study and absolute BA) of ciprofloxacin when co-administered with preparations containing different metallic ions $(1-9,24)$. Relatively high variability in drug bioavailability is evident, both in the pharmacokinetic studies following oral administration of ciprofloxacin hydrochloride tablets alone as well as in the interaction studies in which various drug products or dietary supplements containing different metallic compounds were co-administered. The in vivo data indicate rapid and relatively variable drug absorption with the absolute bioavailability values ranging from 52 to $90 \%$ (average approx. $70 \%$ ). Ciprofloxacin bioavailability in the presence of different metallic compounds was reduced to a variable extent depending mainly on the type of metallic compound/ preparation administered, its dose and dosage regimen. Ciprofloxacin absorption was greatly impaired when it was administered with a high dose of aluminium hydroxide $(1.8 \mathrm{~g})$ or aluminium/magnesium antacid $(1.32 \mathrm{~g} / 1.17 \mathrm{~g}$ and $9 \mathrm{~g} / 6 \mathrm{~g})(2,6,24)$. The extent of ciprofloxacin absorption was reduced by approximately $40 \%$ in the presence of calcium compared to the control reported in specific individual studies $(2,8,9)$ and by $40-80 \%$ in the presence of different iron preparations $(1,3-5,7)$. Multivitamins with zinc, used in the studies of Kara et al. (3), contained a mixture of different metallic compounds, which could have contributed to the observed interaction. Reduced bioavailability observed in a number of in vivo studies following ciprofloxacin co-administration with different metallic ion containing preparations may result from the reduced permeability or reduced solubility of the interaction adduct.

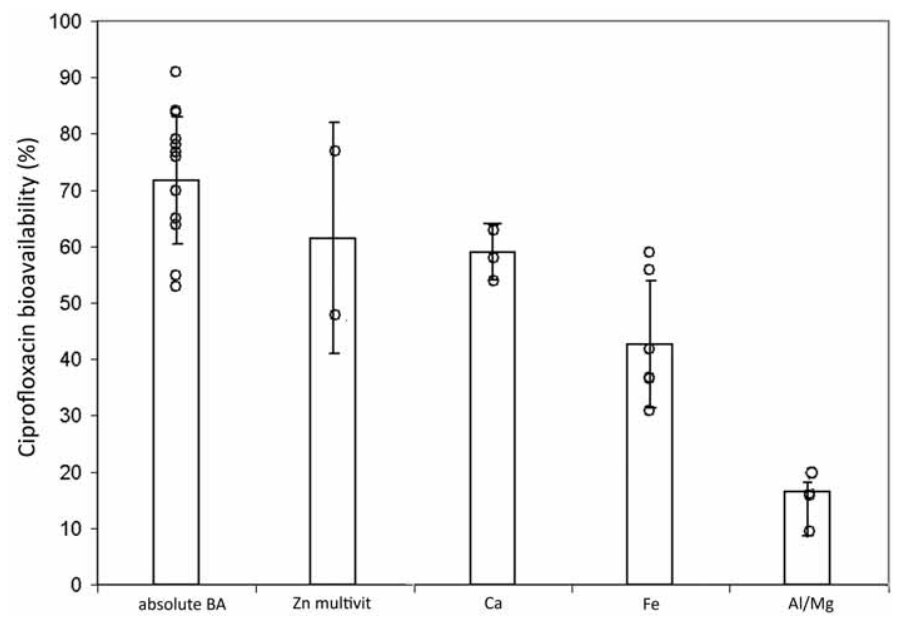

Fig. 1. Ciprofloxacin bioavailability with and without metal ion containing preparations co-administration: summary of available literature data $(1-9,18-24)$. Absolute BA denotes to absolute bioavailability of ciprofloxacin tablets; "Zn multivit, $\mathrm{Ca}, \mathrm{Fe}, \mathrm{Al} / \mathrm{Mg}$," denote relative bioavailability (i.e. ratio of $A U C$ s relevant to the interaction study and "control") of ciprofloxacin when co-administered with preparations containing different metallic ions; column represents the mean values \pm SD (shown as error bars) from a multiple studies represented by open circles). 


\section{Solubility study}

The results of ciprofloxacin hydrochloride solubility studies in water and reactive media containing different amounts of metallic compounds are presented in Fig. 2. Ciprofloxacin hydrochloride solubility in water was $42.0 \pm 0.07 \mathrm{mg} \mathrm{mL}^{-1}$ (i.e., $109 \mathrm{mmol} \mathrm{L}^{-1}$; final $\mathrm{pH}$ 4.04). The amount of ciprofloxacin hydrochloride in all the reactive media containing metallic compounds (i.e. aluminium hydroxide and calcium carbonate suspensions and ferrous sulphate and zinc sulphate solutions) was significantly reduced compared to the amount of ciprofloxacin hydrochloride dissolved in water. Interaction with calcium carbonate led to the most pronounced effect, with a 300-fold decrease in the amount of ciprofloxacin dissolved $(p=0.001)$. Addition of calcium carbonate resulted in a markedly increased $\mathrm{pH}$ value of investigated samples $(\mathrm{pH}>6)$. In the case of zinc sulphate and ferrous sulphate, a similar effect on the amount of ciprofloxacin hydrochloride dissolved was observed and there was no statistically significant difference between the effects of zinc sulphate and ferrous sulphate $(p=0.845)$ on the amount of the drug dissolved. The associated media $\mathrm{pH}$ values were in the same range $(\mathrm{pH} 2.4-3.4)$, indicating that the effect observed was not just a $\mathrm{pH}$ related effect. Addition of insoluble aluminium hydroxide resulted in the least effect on ciprofloxacin hydrochloride dissolution $(p=0.04)$, with a slight increase of media $\mathrm{pH}(\mathrm{pH} 4.8)$.

\section{Dissolution study}

Ciprofloxacin tablet dissolution profiles in water containing different amounts of aluminium hydroxide, calcium carbonate, zinc sulphate and ferrous sulphate are presented in Fig. 3a-d. Ciprofloxacin hydrochloride dissolution from the tablet in water was rapid

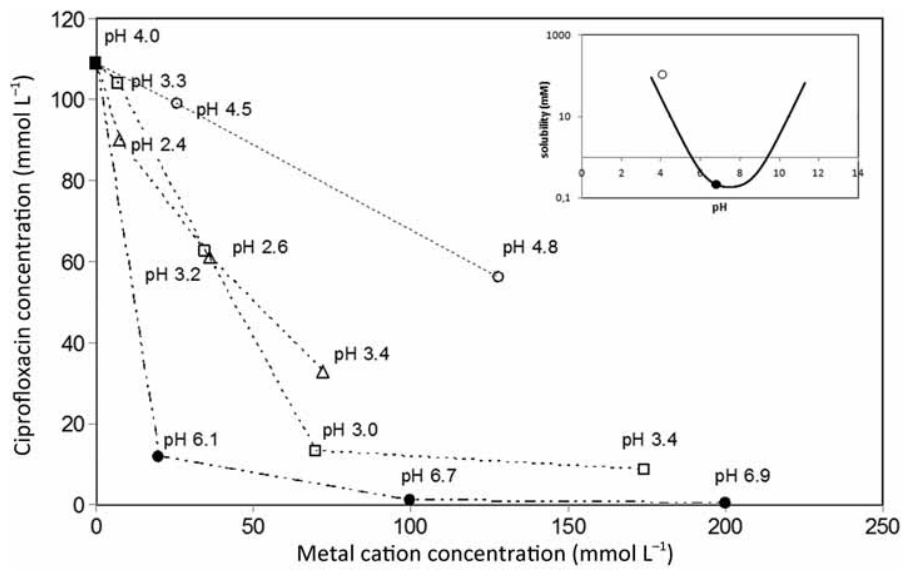

Fig. 2. Ciprofloxacin hydrochloride concentration in media containing different amounts of metallic ion containing compounds: zinc sulfate $(\square)$, ferrous sulfate $(\triangle)$, calcium carbonate $(\bullet)$ and aluminium hydroxide $(\bigcirc)$. Inset: theoretical $\mathrm{pH}$ solubility profile of ciprofloxacin with the data points representing experimentally obtained ciprofloxacin solubility in water (closed circle refers to ciprofloxacin base and open circle to ciprofloxacin $\mathrm{HCl}$ ). 
a)

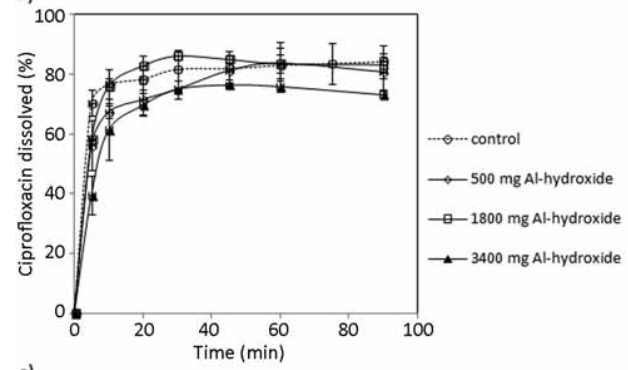

c)

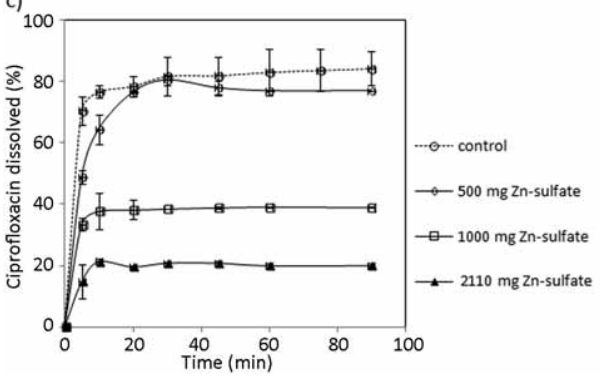

b)

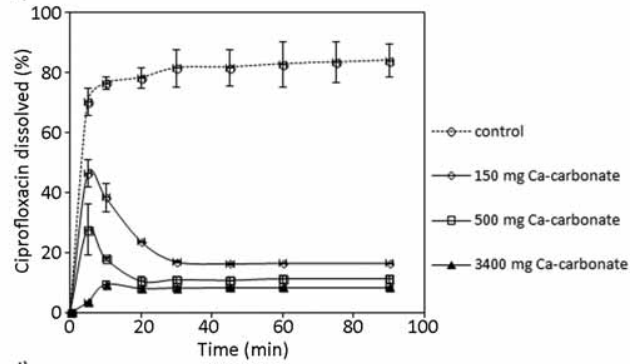

d)

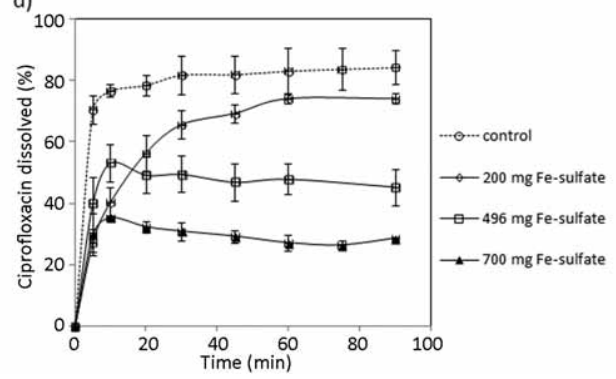

Fig. 3. Ciprofloxacin tablet dissolution in water without (control) and with different amounts of: a) aluminium hydroxide, b) calcium carbonate, c) zinc sulfate and d) ferrous sulfate addition.

and almost complete (final $\mathrm{pH}$ 3.99). In the presence of metallic compounds, relatively fast initial dissolution was observed, followed by the plateau phase after 10 or 20 minutes of investigation. However, the amount of drug dissolved in the "plateau" phase differed markedly in dependence on the type and amount of metallic compound added.

Ciprofloxacin tablet dissolution in the presence of aluminium hydroxide (Fig. 3a) was generally not affected, irrespective of the amount of the hydroxide added (i.e., final $\mathrm{pH}$ ranged from 4.4-4.9). Aluminium hydroxide did not dissolve in water and the excess solid phase preventing tablet dissolution might be responsible for slower ciprofloxacin dissolution from tablets observed at the highest dose of hydroxide added (i.e., $3400 \mathrm{mg}$; media $\mathrm{pH} 4.9$ ).

Calcium carbonate addition had the most pronounced effect on ciprofloxacin hydrochloride solubility and tablet dissolution, associated with the substantial increase in media $\mathrm{pH}$. Upon the addition of poorly soluble calcium carbonate, the initially fast drug dissolution was followed by a notable reduction in the amount of drug dissolved, reaching a maximum of $\sim 20 \%$ (Fig. 3b). Ciprofloxacin dissolution profiles in the presence of different amounts of calcium carbonate exhibited obvious phase transformation during the first 30 minutes of investigation. In this case, the media $\mathrm{pH}$ value increased, resulting in pH 7.2 in media containing 500 and $3400 \mathrm{mg}$ calcium carbonate. Ciprofloxacin concentration in the dissolution media containing the highest dose of calcium carbonate was $0.83 \mathrm{mg} \mathrm{mL}^{-1}$, which was close to the solubility of ciprofloxacin base.

Increased amounts of soluble zinc sulphate and ferrous sulphate in dissolution media resulted in markedly reduced ciprofloxacin tablet dissolution, with 20 and $26 \%$ 
ciprofloxacin dissolved at the highest doses of the investigated metallic compounds, respectively. The final media $\mathrm{pH}$ values obtained in the presence of 500-2110 $\mathrm{mg}$ of zinc sulphate (Fig. 3c) and 200-700 mg of ferrous sulphate (Fig. 3d) ranged from 3.4-3.7 and from 3.1-3.2, respectively, indicating that the effects observed could not be attributed solely to the medium $\mathrm{pH}$ value.

\section{Solid state characterisation}

Results of the powder XRD and FTIR analysis of the solid phases collected from the dissolution vessels after performing ciprofloxacin hydrochloride tablet dissolution in the presence of different metallic compounds are presented in Fig. 4 and 5, respectively. The results obtained indicate that, in the case of reactive media containing aluminium hydroxide, only excess aluminium hydroxide was found. XRD, DSC and FTIR data obtained from the solid phase collected after ciprofloxacin tablet dissolution in the presence of aluminium hydroxide were similar to data reported in the literature $(26,27)$. This result is consistent with the almost complete ciprofloxacin dissolution observed. Literature data report that ciprofloxacin aluminium complexes are more soluble than the parent drug (28). Ciprofloxacin solubility and drug dissolution from the tablet in the presence of aluminium hydroxide were generally not affected, indicating that no ciprofloxacin-aluminium complex was formed. The results of solid state characterisation revealed that the solid phase that crystallized from the media containing calcium carbonate was a hydrate of ciprofloxacin base, which is more than 400-fold less soluble when compared to its hydrochloride salt (29). The X-ray diffractogram of the solid phase collected from ciprofloxacin tablet dissolution in the presence of calcium carbonate was consistent with that of the ciprofloxacin 1:3.7 hydrate (29). FTIR spectra exhibited characteristic bands at 1623, 1580 and $1380 \mathrm{~cm}^{-1}$ assigned to the ketone $\mathrm{C}=\mathrm{O}$ stretch, antisymmetric and symmetric vibrations of the carboxylate anion, respectively, consistent with the spectra found by Dorofeev (30). Thermal analysis of the solid phase collected from ciprofloxacin hydrochloride tablet dissolution in the presence of calcium carbonate revealed a major

Fig. 4. XRD scans of: a) ciprofloxacin base, b) ciprofloxacin $\mathrm{HCl}$ monohydrate and solid phase collected from ciprofloxacin tablet dissolution study in media containing: c) aluminium hydroxide, d) calcium carbonate, e) zinc sulfate and f) ferrous sulfate.

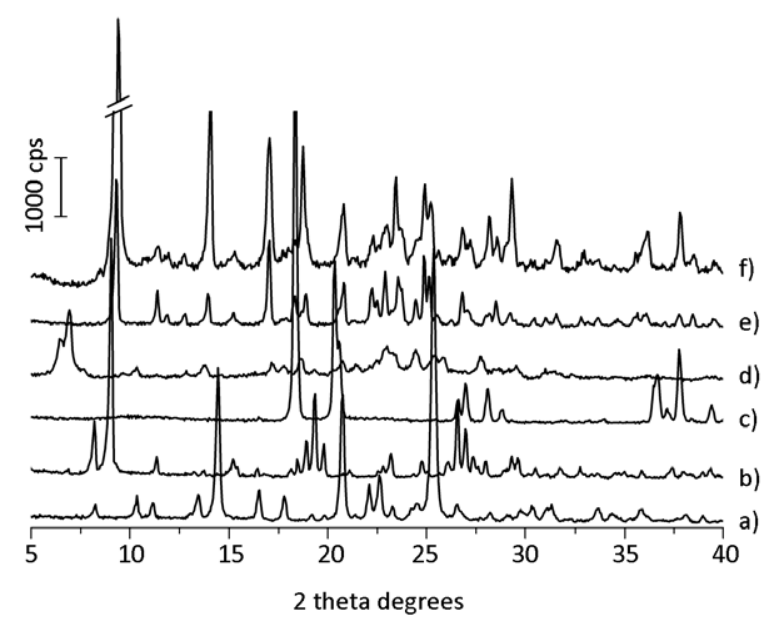




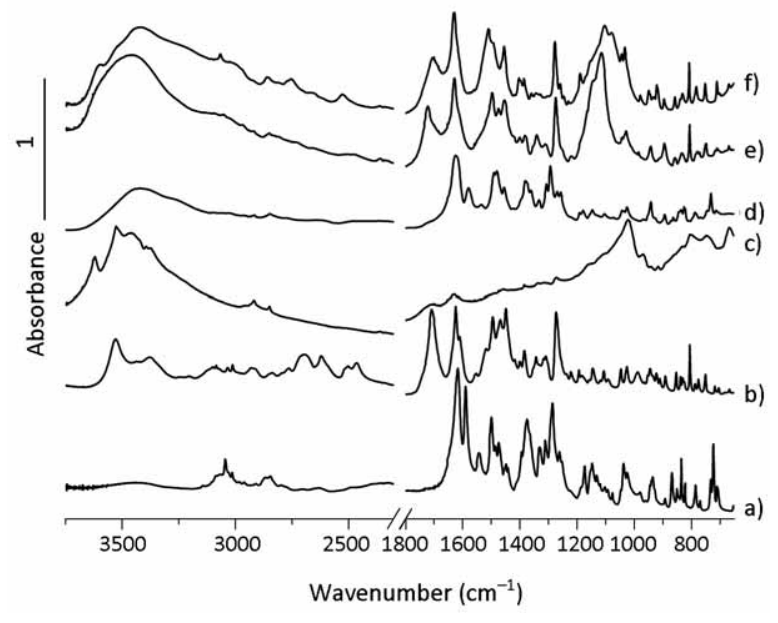

Fig. 5. FTIR scans of: a) ciprofloxacin base, b) ciprofloxacin $\mathrm{HCl}$ monohydrate and solid phase collected from ciprofloxacin tablet dissolution study in media containing: c) aluminium hydroxide, d) calcium carbonate, e) zinc sulfate and f) ferrous sulfate.

endothermic event at approximately $270{ }^{\circ} \mathrm{C}$ preceded by a broad endotherm at $100{ }^{\circ} \mathrm{C}$. The endotherm at $100{ }^{\circ} \mathrm{C}$ was accompanied by a mass loss of approximately $14.5 \%$ and attributed to adsorbed/crystalline water evaporation. Obvious phase transition observed in the dissolution study and caused by increasing $\mathrm{pH}$ media indicated formation of a less soluble product. Having in mind the $\mathrm{pH}$ dependent solubility of ciprofloxacin hydrochloride that precipitates at $\mathrm{pH}$ about 6.8 , formation of the less soluble neutral/zwitterion ciprofloxacin base was expected.

Results of the powder XRD, FTIR and DSC analyses of the solid phase collected from ciprofloxacin tablet dissolution in the presence of ferrous sulphate were described in a previous study (14).

The X-ray diffractogram of the solid phase obtained from ciprofloxacin hydrochloride tablet dissolution in the presence of zinc sulphate shows peaks at $\sim 9$ and $172 \theta$ degrees, the diffractogram was different from those of the ciprofloxacin base and hydrochloride salt (Fig. 4). Thermogravimetry of the solid phase collected from ciprofloxacin tablet dissolution in the presence of zinc sulphate showed an $18.8 \%$ mass loss. FTIR scan of the collected solid phase from ciprofloxacin tablet dissolution in the presence of zinc sulphate exhibited an absorption band at approximately $3500 \mathrm{~cm}^{-1}$, most likely of the $\mathrm{OH}$ stretch vibrations of water molecules. The characteristic band for the $(\mathrm{C}=\mathrm{O})$ vibration of carboxylic and ketone groups in ciprofloxacin hydrochloride monohydrate were at 1709 and $1624 \mathrm{~cm}^{-1}$, respectively. Absorption bands for the $(\mathrm{C}=\mathrm{O})$ vibration of the carboxylic and ketone groups were observed at 1722 and $1629 \mathrm{~cm}^{-1}$ and bands were slightly shifted in comparison with ciprofloxacin hydrochloride (Fig. 5). Absorption band for $\mathrm{C}=\mathrm{O}$ vibration of the carboxylic group was very weak, probably indicating that the $\mathrm{COOH}$ group was deprotonated.

Ciprofloxacin solubility and dissolution from the tablet in the presence of ferrous sulphate and zinc sulphate were reduced to a similar extent. Initially fast drug dissolution was followed by a notable reduction in the amount of drug dissolved, indicating formation of a new ionic species. It was found in the previous study (14) that ciproflo- 
A. Stojković et al:: Biopharmaceutical characterisation of ciprofloxacin-metallic ion interactions: Comparative study into the effect of aluminium, calcium, zinc and iron on drug solubility and dissolution, Acta Pharm. 64 (2014) 77-88.

xacin hydrochloride interaction with ferrous sulphate resulted in the formation of a low solubility complex with the probable chemical structure of $\mathrm{Fe}\left(\mathrm{SO}_{4}\right)_{2}(\mathrm{Cl})_{2}$ (ciprofloxacin $)_{2}$ $\times n \mathrm{H}_{2} \mathrm{O}$, where $n$ is up to 12 molecules of water. Results of the zinc assay (i.e., zinc content $4.0 \%$ ), along with FTIR analysis of the solid phase generated in the interaction media containing zinc sulphate, suggested also complex formation. A close examination of the XRD and FTIR scans of zinc and iron complexes (presented in Figs. 4 and 5) revealed that their structures are likely to be isotypic, implying that the two complexes should have a very similar chemical form. Indeed, based on the chloride and sulphate content analysis (chloride and sulphate contents were 4.5 and $11.7 \%$, respectively), the possible stoichiometry of the zinc complex could be $\mathrm{Zn}\left(\mathrm{SO}_{4}\right)_{2}(\mathrm{Cl})_{2}$ (ciprofloxacin $)_{2} \times n \mathrm{H}_{2} \mathrm{O}$, where $n$ is up to 12 molecules of water. Turel et al. (15) found that the zinc complex with norfloxacin $(\mathrm{nfH}),\left(\mathrm{nfH}_{3}\right)\left(\mathrm{nfH}_{2}\right)\left[\mathrm{ZnCl}_{4}\right] \mathrm{Cl} \times \mathrm{H}_{2} \mathrm{O}$, was isotypic to the copper/norfloxacin compound; thus the occurrence of isotypism for fluoroquinolone-metal complexes is not unexpected. The zinc-ciprofloxacin adduct isolated and presented in this work is new and its structure has been unpublished to date and is also different from the zinc-ciprofloxacin complex with a structure of [ciprofloxacin $\left.\mathrm{H}_{2}\right]_{2} \times\left[\mathrm{ZnCl}_{4}\right] \times \mathrm{H}_{2} \mathrm{O}$ isolated by Zupančič at al. (31).

New solid phases were isolated from the interaction mixtures containing relatively high concentrations of ciprofloxacin hydrochloride and ferrous sulphate, or zinc sulphate. These results indicate that small volume dissolution experiments may be useful for biorelevant dissolution tests, which is in accord with the findings of other authors $(32,33)$.

\section{CONCLUSIONS}

Ciprofloxacin hydrochloride metallic ion in vitro interaction studies revealed that drug solubility and dissolution were impaired to different extents in dependence on the type of the metallic compound present. Based on the quantitative analysis and FTIR spectra, precipitates collected after drug dissolution studies in the media containing zinc sulphate and ferrous sulphate were identified as poorly soluble complexes. Analysis of the novel zinc complex isolated in this work is consistent with stoichiometry of $\mathrm{Zn}\left(\mathrm{SO}_{4}\right)_{2}(\mathrm{Cl})_{2}$ (ciprofloxacin $)_{2} \times n \mathrm{H}_{2} \mathrm{O}$, where $n$ is up to 12 molecules of water. Such data are in accord with our earlier findings on the probable chemical structure of the ciprofloxacin-iron interaction product. In contrast, interaction studies with aluminium hydroxide and calcium carbonate did not result in complex formation. Ciprofloxacin hydrochloride salt was identified upon evaporation of the dissolution media in interaction studies with aluminium hydroxide, while the interaction with calcium carbonate resulted in precipitation of ciprofloxacin (base) hydrate. Since ciprofloxacin exhibits an absorption window in the proximal small intestine, it may be postulated that drug co-administration with a metallic compound containing preparations may result in different types of interactions that lead to reduced ciprofloxacin hydrochloride solubility and subsequent absorption.

Acknowledgements. - A part of the work was performed under Project TR-34007 supported by the Ministry of Education, Science and Technological Development, Republic of Serbia. Lidia Tajber, Krzysztof J. Paluch, and Owen Corrigan acknowledge the support to this research of the Solid State Pharmaceutical Cluster, supported by the Science Foundation Ireland under grant number 07/SRC/B1158. 
A. Stojković et al.: Biopharmaceutical characterisation of ciprofloxacin-metallic ion interactions: Comparative study into the effect of aluminium, calcium, zinc and iron on drug solubility and dissolution, Acta Pharm. 64 (2014) 77-88.

\section{REFERENCES}

1. J. R. Brouwers, H. J. Van der Kam, J. Sijtsma and J. H. Proost, Decreased ciprofloxacin absorption with concomitant administration of ferrous fumarate, Pharm. Weekbl. Sci. 12 (1990) 182-183; DOI: $10.1007 /$ BF01980042.

2. R. W. Frost, J. T. Lettieri, A. Noe, E. C. Shamblen and K. Lasseter, Effect of aluminium hydroxide and calcium carbonate antacids on ciprofloxacin bioavailability, Antimicrob. Agents Chemother. 36 (1992) 830-832; DOI: 10.1128/AAC.36.4.830.

3. M. Kara, B. B. Hasinoff, W. D. McKay and R. C. N. Campbell, Clinical and chemical interactions between iron preparations and ciprofloxacin, Brit. J. Clin. Pharmacol. 31 (1991) 257-261; DOI: 10.1111/j.1365-2125.1991.tb05526.x.

4. P. Lehto, T. K. Kivisto and J. P. Neuvonen, The effect of ferrous sulphate on the absorption of norfloxacin, ciprofloxacin and ofloxacin, Brit. J. Clin. Pharmacol. 37 (1994) 82-85; DOI: 10.1111/ j.1365-2125.1994.tb04245.x.

5. R. E. Polk, D. P. Healy, J. Sahai, L. Drwal and E. Racht, Effect of ferrous sulfate and multivitamins with zinc on absorption of ciprofloxacin in normal volunteers, Antimicrob. Agents Chemother. 33 (1989) 1841-1844; DOI: 10.1128/AAC.33.11.1841.

6. D. E. Nix, W. A. Watson, M. E. Lener, R. W. Frost, G. Krol, H. Goldstein, J. Lettieri and J. J. Schentag, Effects of aluminum and magnesium antacids and ranitidine on the absorption of ciprofloxacin, Clin. Pharmacol. Ther. 46 (1989) 700-705; DOI: 10.1038/clpt.1989.207.

7. E. Pazzucconi, S. Barbi, D. Baldassarre, N. Colombo, F. Dorigotti and C. R. Sirtori, Iron-ovotransferrin preparation does not interfere with ciprofloxacin absorption, Clin. Pharmacol. Ther. 59 (1996) 418-422; DOI: 10.1016/S0009-9236(96)90110-1.

8. A. L. Neuhofel, J. H. Wilton, J. M. Victory, L. G. Hejmanowsk and G. W. Amsden, Lack of bioequivalence of ciprofloxacin when administered with calcium-fortified orange juice: a new twist on an old interaction, J. Clin. Pharmacol. 42 (2002) 461-466; DOI: 10.1177/00912700222011391.

9. J. Sahai, D. P. Healy, J. Stotka and R. E. Polk, The influence of chronic administration of calcium carbonate on the bioavailability of oral ciprofloxacin, Brit. J. Clin. Pharmacol. 35 (1993) 302-304.

10. F. Kozjek, E. Palka, I. Krizman and P. Vodopivec, Pharmacokinetics of ciprofloxacin metal complexes, Acta Pharm. 46 (1996) 109-114.

11. S. Rodriguez Cruz, I. Gonzalez Alonso, A. Sanchez-Navarro and L. Sayalero Marinero, In vitro study of the interaction between quinolones and polyvalent cations, Pharm. Acta Helv. 73 (1999) 237-245; DOI: 2048/10.1016/S0031-6865(98)00029-6.

12. S. Žakelj, K. Berginc, D. Uršić, M. Veber and A. Kristl, Metal cation-fluoroquinolone complexes do not permeate through the intestinal absorption barrier, J. Pharm. Biomed. Anal. 53 (2010) 655-659; DOI: $10.1016 / j . j p b a .2010 .05 .021$.

13. K. Papai, M. Budai, K. Ludanyi, I. Antal and I. Klebovich, In vitro food-drug interaction study: Which milk component has a decreasing effect on the bioavailability of ciprofloxacin? J. Pharm. Biomed. Anal. 52 (2010) 37-42; DOI: 10.1016/j.jpba.2009.12.003.

14. J. Parojčić, A. Stojković, L. Tajber, S. Grbić, K. Paluch, Z. Đurić and O. I. Corrigan, Biopharmaceutical characterization of ciprofloxacin HCl-ferrous sulfate interaction, J. Pharm. Sci. 100 (2011) 5174-5184; DOI: 10.1002/jps.22707.

15. I. Turel, The interactions of metal ions with quinolone antibacterial agents, Coord. Chem. Rev. 232 (2002) 22-47; DOI: 10.1016/S0010-8545(02)00027-9.

16. A. K. Chattah, Y. G. Linck, G. A. Monti, P. R. Levstein, S. A. Breda, R. H. Manzo and M. E. Olivera, NMR and IR characterization of the aluminium complexes of norfloxacin and ciprofloxacin fluoroquinolones, Magn. Reson. Chem. 45 (2007) 850-859; DOI: 10.1002/mrc.2058.

17. L. Tajber, D. O. Corrigan, O. I. Corrigan and A. M. Healy, Spray drying of budesonide, formoterol fumarate and their composites - I. Physicochemical characterization, Int. J. Pharm. 367 (2009) 79-85; DOI: 10.1016/j.ijpharm.2008.09.030. 
A. Stojković et al.: Biopharmaceutical characterisation of ciprofloxacin-metallic ion interactions: Comparative study into the effect of aluminium, calcium, zinc and iron on drug solubility and dissolution, Acta Pharm. 64 (2014) 77-88.

18. T. Bergan, S. B. Thorsteinsson, I. M. Kolstad and S. Johnsen, Pharmacokinetics of ciprofloxacin after intravenous and increasing oral doses, Eur. J. Clin. Microbiol. 5 (1986) 187-192; DOI: $10.1007 /$ BF02013984.

19. K. Borner, G. Hoffken, H. Lode, P. Koeppe, C. Prinzing, P. Glatzel, R. Wiley, P. Olschewski, B. Sievers and D. Reinitz, Pharmacokinetics of ciprofloxacin in healthy volunteers after oral and intravenous administration, Eur. J. Clin. Microbiol. 5 (1986) 179-186; DOI: 10.1007/BF02013983.

20. G. L. Drusano, H. C. Standiford, K. Plaisance, A. Forrest, J. Leslie and J. Caldwell, Absolute oral bioavailability of ciprofloxacin, Antimicrob. Agents Chemother. 30 (1986) 444-446; DOI: 10.1128/ AAC.30.3.444.

21. G. Hoffken, H. Lode, C. Prinzing, K. Borner and P. Koeppem, Pharmacokinetics of ciprofloxacin after oral and parenteral administration, Antimicrob. Agents Chemother. 27 (1985) 375-379; DOI: 10.1128/AAC.27.3.375.

22. J. T. Lettieri, M. C. Rogge, L. Kaiser, R. M. Echols and A. H. Heller, Pharmacokinetic profiles of ciprofloxacin after single intravenous and oral doses, Antimicrob. Agents Chemother. 36 (1992) 993-996; DOI: 10.1128/AAC.36.5.993.

23. K. I. Plaisance, G. L. Drusano, A. Forrest, C. I. Bustamante and H. C. Standiford, Effect of dose size on bioavailability of ciprofloxacin, Antimicrob. Agents Chemother. 131 (1987) 956-958; DOI: 10.1128/ AAC.31.6.956.

24. H. Lode, Drug interactions with quinolones, Clin. Infect. Dis. 10 (1988) S132-S136; DOI: 10.1093/ clinids/10.Supplement_1.S132.

25. S. Harder, U. Fuhr, D. Beermann and A. H. Staib, Ciprofloxacin absorption in different regions of the human gastrointestinal tract. Investigations with hf-capsule, Brit. J. Clin. Pharmacol. 30 (1990) 35-39; DOI: 10.1111/j.1365-2125.1990.tb03740.x.

26. H. Kathyayini, I. Willems, A. Fonseca, J. B. Nagy and N. Nagaraju, Catalytic materials based on aluminium hydroxide, for the large scale production of bundles of multi-walled (MWNT) carbon nanotubes, Catal. Commun. 7 (2006) 140-147; DOI: 10.1016/j.catcom.2005.05.010.

27. S. Qing Su, J. Yang, H. W. Ma, F. Jiang, Y. Q. Liu and G. Li, Preparation of ultrafine aluminum hydroxide from coal fly ash by alkali dissolution process, Integr. Ferroelectr. 128 (2011) 155-162; DOI: $10.1080 / 10584587.2011 .576626$.

28. S. A. Breda, A. F. Jimenez-Kairuz, R. H. Manzo and M. E. Olivera, Solubility behavior and biopharmaceutical classification of novel high-solubility ciprofloxacin and norfloxacin pharmaceutical derivatives, Int. J. Pharm. 371 (2009) 106-113; DOI: 10.1016/j.ijpharm.2008.12.026.

29. L. Mafra, S. M. Santos, R. Siegel, I. Alves, F. A. Almeida Paz, D. Dudenko and H. W. Spiess, Packing interactions in hydrated and anhydrous forms of the antibiotic ciprofloxacin: a solid-state NMR, X-ray diffraction, and computer simulation study, J. Am. Chem. Soc. 134 (2012) 71-74; DOI: 10.1021/ja208647n.

30. V. L. Dorofeev, The betaine like structure and infrared spectra of drugs of the fluoroquinolone group, Pharm. Chem. J. 38 (2004) 698-702; DOI: 10.1007/s11094-005-0064-5.

31. M. Zupančić, I. Turel, P. Bukovec, A. J. P. White and D. J. Williams, Synthesis and characterization of two novel zinc(II) complexes with ciprofloxacin crystal structure of $\left[\mathrm{C}_{17} \mathrm{H}_{19} \mathrm{~N}_{3} \mathrm{O}_{3} \mathrm{~F}\right]_{2}$ $\mathrm{ZnCl}_{4} 2 \mathrm{H}_{2} \mathrm{O}$, Croat. Chem. Acta. 74 (2001) 61-74.

32. R. Takano, N. Takata, R. Saito, K. Furumoto, S. Higo, Y. Hayashi, M. Machida, Y. Aso and S. Yamashita, Quantitative analysis of the effect of supersaturation on in vivo drug absorption, Mol. Pharm. 7 (2010) 1431-1440; DOI: 10.1021/mp100109a.

33. E. Scheubel, M. Lindenberg, E. Beyssac and J. M. Cardot, Small volume dissolution testing as a powerful method during pharmaceutical development, Pharmaceutics 2 (2010) 351-363; DOI: 10.3390 / pharmaceutics2040351. 